\title{
Cirugía valvular reparadora en endocarditis infecciosa
}

\author{
Ricardo Zalaquett S, Luis Garrido 0 , Francisca Casas R, \\ Sergio Morán V, Manuel J Irarrázaval LI, Pedro Becker R, \\ Sandra Braun J, Samuel Córdova A, G astón Chamorro S, \\ Iván G odoy J, Fernando Yáñez D, Jaime Labarca L, \\ Carlos Pérez C.
}

\section{Valve repair surgery in infectious endocarditis}

Background: Valve replacement has been the treatment of choice for patients with valvular complications of infectious endocarditis (IE). However, excellent results with valve repair allowed it to become a new therapeutic alternative for these patients. Aim: To evaluate the results of valve repair in patients with valvular complications of IE. Patients and Methods: From January 1991 to December 2000, 14 patients with valvular complications of IE underwent valve repair. Mean age was $37.9 \pm 14.9$. Results: New York Heart Association (NYHA) class was $2.8 \pm 0.9$. IE was located in the aortic in $6(42 \%)$, in the mitral valve in $4(29 \%)$ and in both valves in 4 cases (29\%). Surgical indication was hemodynamic in $50 \%$ of the cases, echocardiographic in $29 \%$ and septic in $21 \%$. Five aortic valves were bicuspid, 3 mitral valves were myxomatous and the rest were normal. The most common septic lesions were vegetations and leaflet perforations. A total of 23 aortic and 21 mitral valve repair procedures were performed. There were no deaths. Only 1 patient had a surgical complication (renal failure and prolonged mechanical ventilation). Follow-up was $100 \%$ complete. There was not late mortality. One patient with bone marrow aplasia required reoperation for a new episode of IE 19 months later. At the end of the follow-up NYHA class was $1.3 \pm 0.6$ and echocardiography showed a mild or absence of valve regurgitation in most patients. Conclusions: Valve repair surgery in IE has good results, with advantages over valve replacement (Rev Méd Chile 2004; 132: 307-15).

(Key Words: Cardiovascular surgical procedures; Endocarditis, bacterial; Surgical procedures, operative)

Recibido el 8 de abril, 2003. Aceptado en versión corregida el 1 de diciembre, 2003.

Departamentos de Enfermedades Cardiovasculares y Medicina Interna, Facultad de Medicina, Pontificia Universidad Católica de Chile

Correspondencia a: Dr. Ricardo Zalaquett S. Marcoleta 367, $6^{\circ}$ piso. Teléfonos: 6333030 - 686 3231. Fax: 6390108.

E-mail: rzalaque@med.puc.cl 
$\int u$ unto al desarrollo de antibióticos de alto poder bactericida, la cirugía ha modificado la historia natural de la endocarditis infecciosa al permitir la erradicación del proceso infeccioso, reparando a la vez el daño tisular y reestableciendo una hemodinamia normal ${ }^{1}$. En la mayoría de los casos, esto ha significado efectuar un reemplazo protésico de la válvula dañada. Sin embargo, tanto las prótesis biológicas como las mecánicas tienen serios inconvenientes. Así, la durabilidad de las primeras es limitada y las segundas requieren anticoagulación de por vida. Además, el reemplazo protésico en presencia de una endocarditis tiene elevada mortalidad y conlleva un riesgo de endocarditis protésica, la que tiene mayor gravedad que la infección de válvula nativa ${ }^{1-4}$.

Por otra parte, las ventajas de la cirugía reparadora de la insuficiencia mitral son conocidas y aceptadas, en especial respecto a mantener la geometría y función del ventrículo izquierdo, además de no requerir anticoagulación y de una menor morbilidad valvular, incluyendo la endocarditis $^{5,6}$. Igualmente, la preservación de la válvula aórtica resulta cada vez más atractiva al no requerir la anticoagulación del paciente, por una parte, $y$, por otra, no producir gran modificación de la raíz aórtica que dificulte eventuales reoperaciones, como lo es el implante de un homo o autoinjerto ${ }^{7,8}$. En ambos casos, además, la ausencia de material protésico tendría menor susceptibilidad a reinfecciones y si éstas se llegasen a producir existiría una mejor probabilidad de curar la infección con tratamiento antibiótico que si existiera una prótesis cuya infección, en general, es indicación de cirugía $1-3,6,9,10$.

Finalmente, en la actualidad, la degeneración mixomatosa de la válvula mitral y la válvula aórtica bicúspide son el principal sustrato para el desarrollo de una endocarditis. Ambas situaciones son hoy susceptibles de una cirugía reparadora, como lo sería el daño tisular producido por una endocarditis ${ }^{1,4,9}$.

El interés en nuestra institución por efectuar cirugía reconstructora de las válvulas cardíacas se inició a comienzos de la década recién pasada y ha sido objeto de diferentes comunicaciones ${ }^{11-15}$. En esta oportunidad, presentamos nuestra experiencia con la cirugía valvular reparadora en endocarditis infecciosa.

\section{PACIENTES Y MÉTODO}

El período en estudio estuvo comprendido entre enero de 1991 y diciembre de 2000, puesto que en 1991 se introdujo en nuestro hospital la cirugía reparadora de la insuficiencia valvular de acuerdo a los principios y técnicas de Carpentier para la válvula mitral y de Cosgrove para la válvula aórtica ${ }^{5,11-14,16}$. En el período señalado, un total de 118 pacientes fueron operados por una endocarditis de la válvula aórtica, mitral o de ambas, de los cuales 14 (12\%) tuvieron una reparación valvular, los que constituyen la base de este estudio. Nueve pacientes (64\%) eran hombres. La edad promedio fue $37,9 \pm 14,9$ años (18 a 66 años). El diagnóstico de endocarditis se basó hasta 1993 en los criterios de Von Reyn y a partir de 1994 se efectuó de acuerdo a los criterios establecidos por Durack y asociados ${ }^{17,18}$. La endocarditis se consideró activa si los hemocultivos eran positivos al momento de la cirugía, los hallazgos intraoperatorios eran compatibles con un proceso inflamatorio agudo y los cultivos o la tinción de Gram del tejido resecado eran positivos. Por el contrario, la endocarditis se consideró inactiva si se había completado el tratamiento antibiótico y los hemocultivos eran negativos al momento de la operación al igual que los cultivos y la tinción de Gram del tejido resecado.

La indicación quinúrgica se efectuó de acuerdo a criterios habituales detallados en nuestras publicaciones previas ${ }^{19,20}$, independientemente de la posibilidad de reparar o no la válvula infectada. En pacientes con endocarditis inactiva, la cinugía se indicó en aquellos con insuficiencia valvular secundaria al proceso infeccioso con repercusión hemodinámica.

Los requisitos para efectuar una reparación valvular en presencia de un proceso infeccioso activo fueron los siguientes: 1) Germen conocido. 2) Sensibilidad antibiótica determinada. 3) Erradicación quirúrgica del tejido infectado. 4) Control ecocardiográfico transesofágico intraoperatorio ${ }^{23}$. Esto último es indispensable para la evaluación final de la calidad funcional de la reparación previo al cierre del esternón.

La información se obtuvo de la revisión de las fichas clínicas y protocolos operatorios. Para el seguimiento, los pacientes fueron contactados durante enero 2001. Igualmente, se revisó el último ecocardiograma disponible luego del alta hospitalaria. 


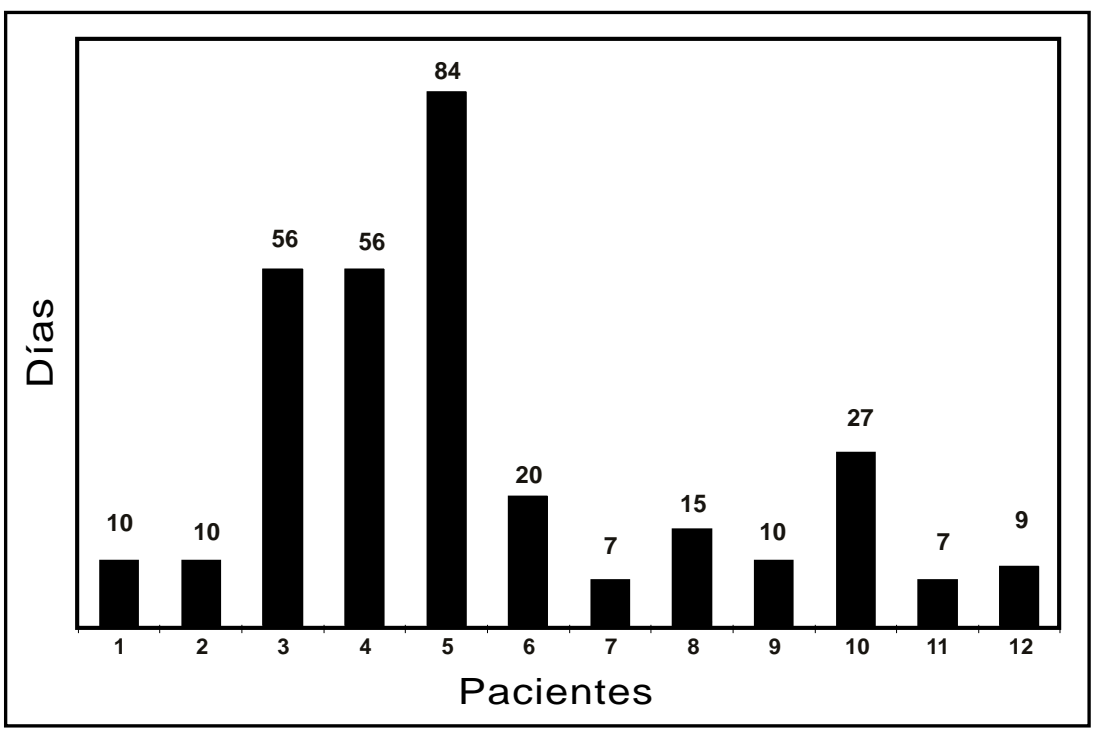

FiguRA 1. Duración del tratamiento antibiótico preoperatorio en los 12 pacientes con endocarditis infecciosa activa. El promedio de días de tratamiento fue de $25,9 \pm$ 25,4 (7 a 84) días para el grupo total. Si se excluye la paciente 5 el promedio de días de tratamiento antibiótico fue de 20,6 $\pm 18,5$ (7 a 56) días (ver texto).

Se efectuó un análisis estadístico descriptivo por lo que los datos se expresan como promedios \pm desviación estándar.

\section{RESUlTADOS}

Característica clínicas. En 12 pacientes (86\%) la endocarditis fue considerada activa de acuerdo a los criterios señalados y en los 2 restantes inactiva. En los casos de endocarditis activa, el tiempo transcumido entre el inicio de los síntomas y la cinugía fue mayor a 2 meses en 8 casos (67\%) y menor de 2 meses en los 4 restantes. La Figura 1 grafica la duración del tratamiento antibiótico preoperatorio en estos 12 pacientes con endocarditis activa. Así, para el total de éstos el promedio de días de tratamiento preoperatorio fue de 25,9 $\pm 25,4$ días con un margen de 7 a 84 días. Sin embargo, si se excluye la paciente 5, la que debutó con una infección pelviana, en el curso de la cual desarrolló una endocarditis, manteniéndose siempre con tratamiento antimicrobiano, el promedio de días de tratamiento antibiótico preoperatorio fue de 20,6 \pm 18,5 días con un margen de 7 a 56 .

La capacidad funcional (CF) preoperatoria promedio fue de $2,8 \pm 0,9$, estando un paciente en $\mathrm{CF}$ I (7\%), 4 en CF II (29\%), 6 en CF III (43\%) y 3 en CF IV (21\%).

La Figura 2 muestra las válvulas comprometidas por la endocarditis. En 6 casos (42\%) la válvula infectada fue la aórtica, en 4 (29\%) la mitral y en los

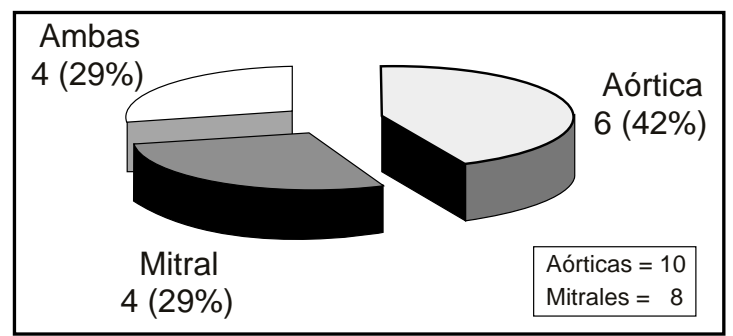

FIGURA 2. Válvulas cardíacas dañadas por endocarditis infecciosa.

otros 4 (29\%) ambas válvulas estaban comprometidas. Así, hubo un total de 10 válvulas aórticas y 8 mitrales dañadas por endocarditis. La ecocardiografía preoperatoria demostró insuficiencia valvular masiva (4+) en 4 válvulas aórticas y en 4 mitrales, insuficiencia significativa (3+) en 4 válvulas aórticas y 1 mitral, insuficiencia moderada (2+) en 2 válvulas aórticas y en 1 mitral e insuficiencia leve (1+) en 2 válvulas mitrales (Figura 3).

La indicación operatoria fue hemodinámica, por insuficiencia cardíaca, en 7 casos (50\%), ecocardiográfica, esto es presencia de grandes vegetaciones, abscesos perianulares o ambas lesiones, en 4 casos (29\%) y séptica, por persistencia de signos de infección activa, en 3 casos (21\%).

Bacteriología. De los 12 pacientes con endocarditis activa, el microorganismo responsable fue Streptococcus viridans en 4, Streptococcus grupo 
D en 3, Staphylococcus aureus en 2, Streptococcus grupo B en 1, Corynebacterium sp en 1 y Haemophilus sp en 1. De los 2 pacientes con endocarditis inactiva, el germen causante del daño valvular había sido Streptococcus viridans en $1 \mathrm{y}$ en el otro no fue identificado.

Hallazgos y procedimientos quirúrgicos. De las 10 válvulas aórticas comprometidas, 5 eran válvulas de 3 velos, esencialmente normales y 5 eran bicúspides. De las 8 válvulas mitrales, 5 eran estructuralmente normales y 3 presentaban cambios mixomatosos. La Figura 4 muestra los hallaz- gos secundarios al proceso infeccioso. Los más frecuentes fueron las vegetaciones y las perforaciones de velos, tanto en válvula mitral como aórtica. Las técnicas reparadoras utilizadas para la endocarditis aórtica consistieron en resección de vegetaciones en 7 casos, resección triangular de velo mayor en 4 casos de válvulas bicúspides, parche con pericardio autólogo en 6 casos de perforación de velos, sutura directa de perforación en un caso y anuloplastia subcomisural en 5 casos. En total, se efectuaron 23 procedimientos reparadores aórticos (Figura 5). Para la endocarditis mitral las técnicas utilizadas consistieron en

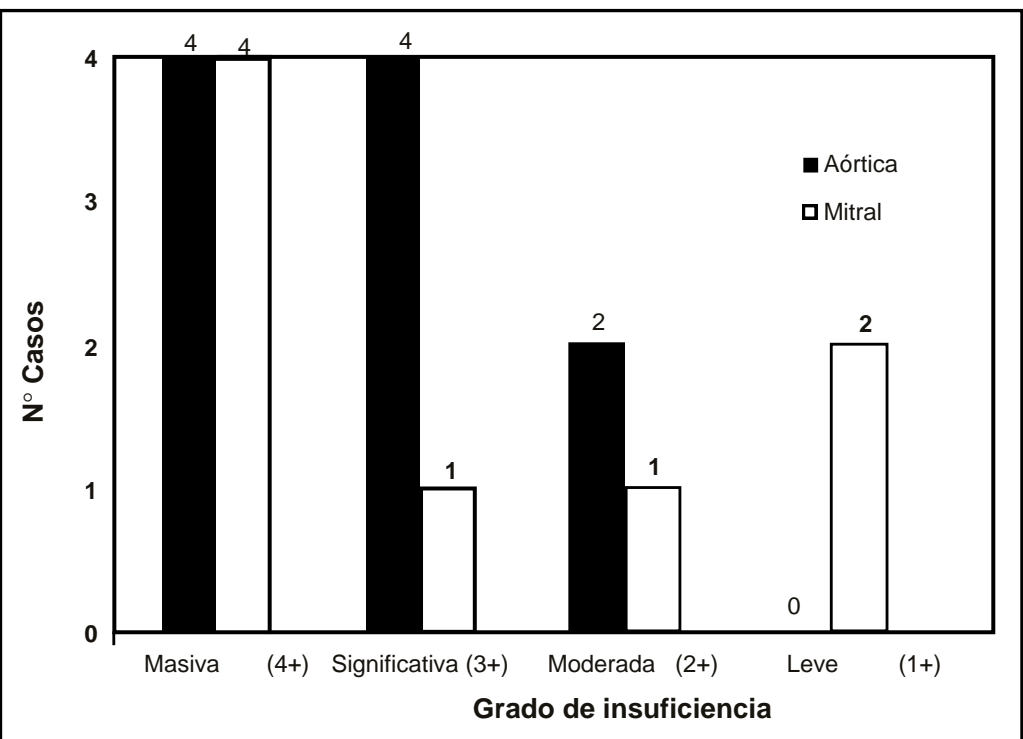

FIgURA 3. Insuficiencia valvular preoperatoria determinada por ecocardiografía transtorácica y transesofágica.

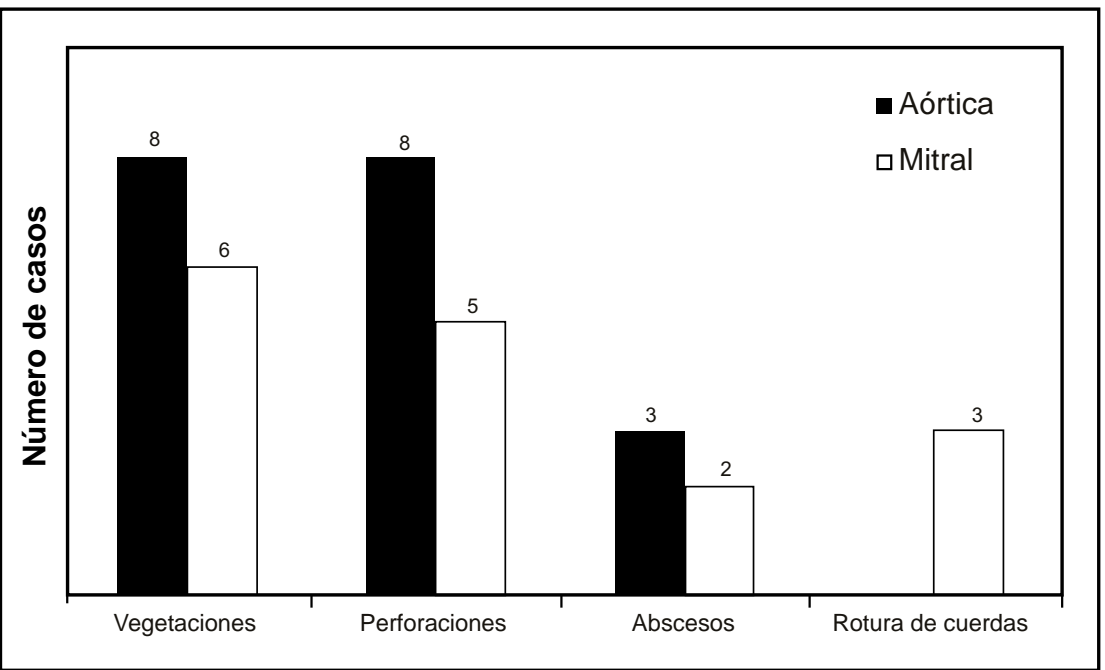

Figura 4. Hallazgos anatomopatológicos intraoperatorios secundarios al proceso infeccioso. 
resección de vegetaciones en 6 casos, resección cuadrilateral del velo posterior en 2 , cierre de perforación con parche de pericardio en 4 , sutura directa de perforación en 2 y transferencia de cuerdas tendíneas desde el velo posterior al anterior en 1 caso. En 6 pacientes la reparación se complementó con una anuloplastia con anillo de Carpentier-Edwards ${ }^{\mathrm{TM}}$. Así, en total se realizaron 21 procedimientos reparadores mitrales (Figura 6). Los abscesos perianulares fueron debridados $\mathrm{y}$ obliterados directamente 0 con un parche de pericardio autólogo.

Mortalidad y morbilidad. No hubo mortalidad intrahospitalaria ni a 30 días de la operación. Sólo una paciente presentó morbilidad relacionada al acto quirúrgico, consistente en ventilación mecánica prolongada e insuficiencia renal transitoria. La indicación quirúrgica de esta paciente con endocarditis mitroaórtica fue sepsis no controlada con embolias múltiples.

Seguimiento. Se completó en $100 \%$ de los casos con promedio de 39,4 $\pm 25,3$ meses y margen de 4 a 78 meses. No hubo mortalidad durante el seguimiento. Un paciente requirió una reoperación, consistente en un recambio aórtico a los 19 meses de su reparación aórtica por una nueva endocarditis presentada en el curso de una aplasia medular secundaria al tratamiento

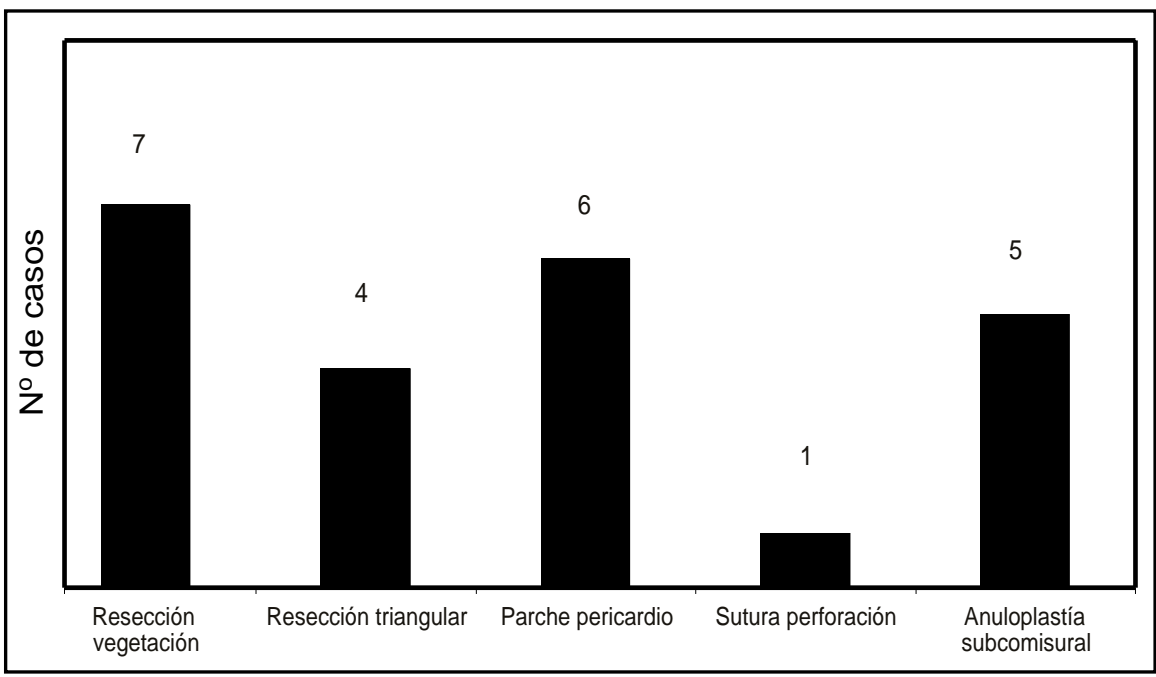

Figura 5. Técnicas quirúrgicas de reparación valvular aórtica. En total se efectuaron 23 procedimientos reparadores valvulares aórticos (ver texto).

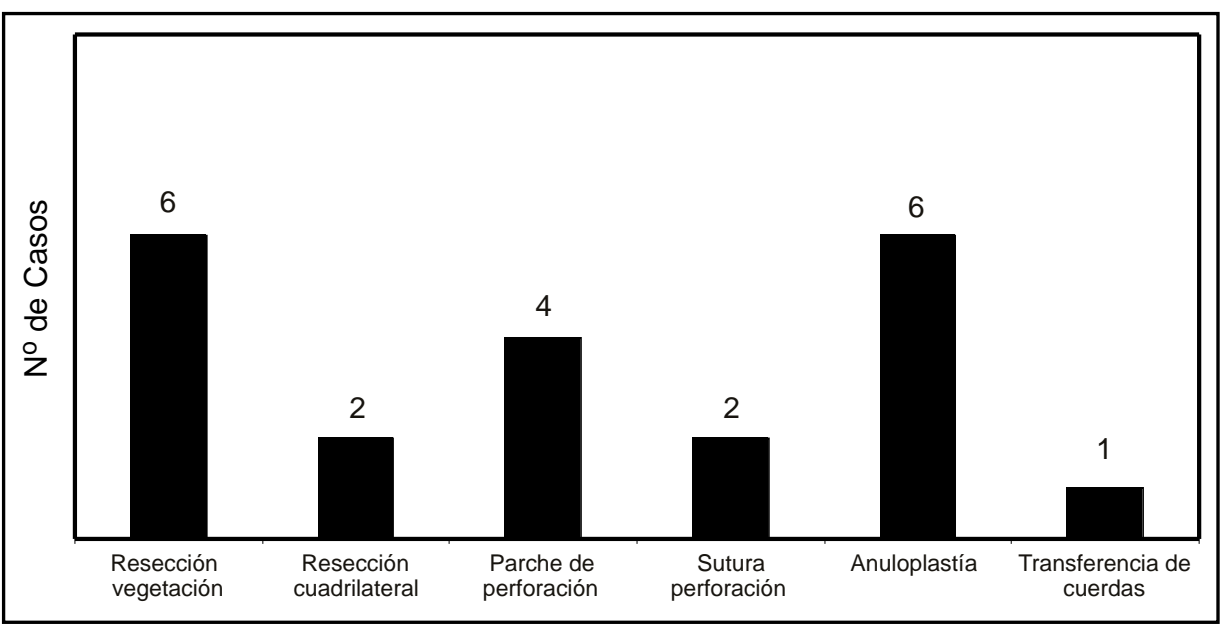

Figura 6. Técnicas quirúrgicas de reparación valvular mitral. En total se realizaron 21 procedimientos reparadores valvulares mitrales (ver texto). 
antibiótico de la primera endocarditis. Este fue el único caso de recidiva de endocarditis. Ningún paciente se encontraba en tratamiento anticoagulante al término del seguimiento, así como no se registró ningún caso de tromboembolismo.

$\mathrm{Al}$ cierre del seguimiento, la $\mathrm{CF}$ promedio fue de $1,3 \pm 0,6$. Nueve pacientes $(64 \%)$ se encontraban en CF I y 5 (36\%) en CF II. La Figura 7 compara la CF pre y post operatoria. Así, la CF promedio para el grupo total disminuyó desde 2,8 $\pm 0,9$ a 1,3 $\pm 0,6$. La ecocardiografía de control (Figura 8) demostró ausencia de insuficiencia en 3 válvulas aórticas y en 5 mitrales, insuficiencia leve (1+) en 4 válvulas aórticas y en 2 mitrales, insuficiencia moderada (2+) en 2 válvulas aórticas y una insuficiencia significativa (3+) en una válvula mitral.

\section{DisCUSIÓN}

Pacientes y método. Esta es una serie limitada de pacientes que representaron a $12 \%$ del total de operados por endocarditis durante el período en estudio. Lo anterior es resultado, en parte, de la curva de aprendizaje quirúrgico en cuanto a las técnicas de reparación valvular y de los cambios de criterio del manejo operatorio de la endocarditis, en especial en relación a los conceptos de erradicación macroscópica del proceso infeccioso y no uso de material protésico ${ }^{1-4}$. También es resultado de la exigencia de conocer el germen responsable de la infección y su sensibilidad antibiótica, lo que sólo en los últimos años se ha logrado conseguir en la mayoría de los $\operatorname{casos}^{23}$. Esto último es necesario para guiar el tratamiento antibiótico postoperatorio.

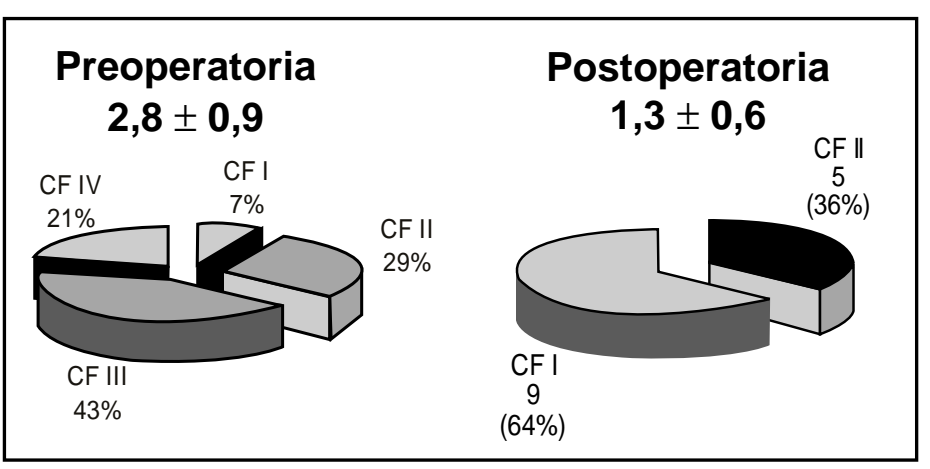

Figura 7. Capacidad funcional pre y post operatoria alejada (ver texto).

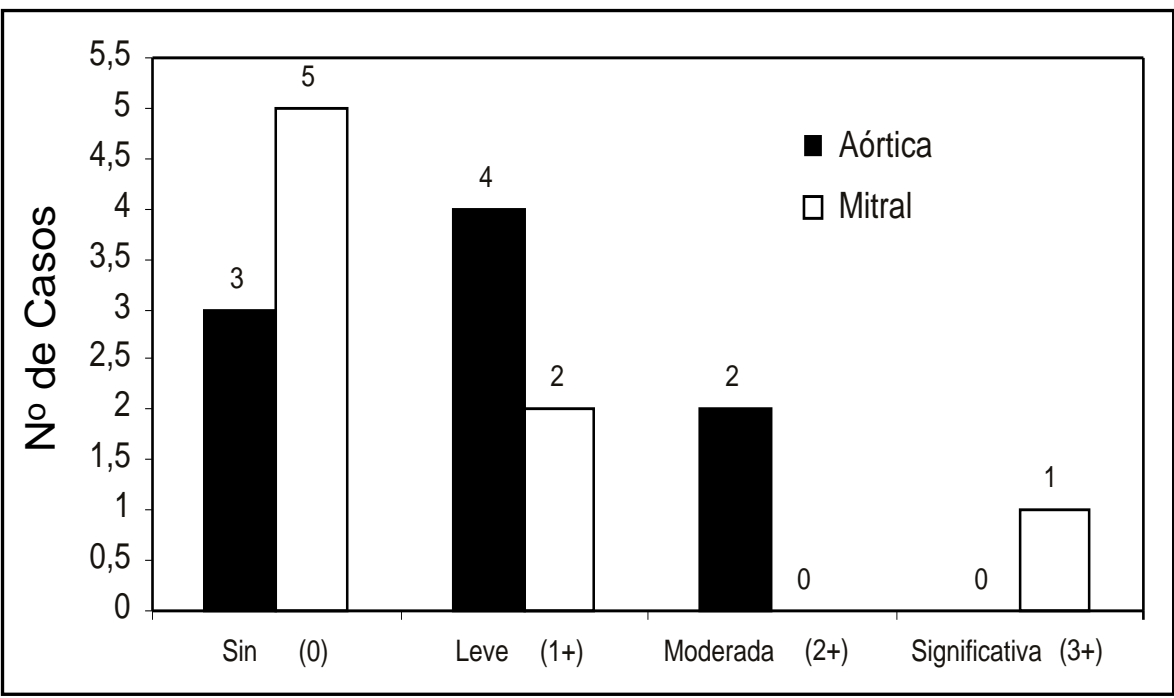

Figura 8. Insuficiencia valvular postoperatoria alejada determinada por ecocardiograma transtorácico (ver texto). 
El trabajo comesponde a un estudio observacional descriptivo en el que, si bien los pacientes fueron prospectivamente ingresados a un registro de reparaciones valvulares, los resultados fueron obtenidos retrospectivamente, por lo que cuenta con todas las limitaciones propias de este tipo de estudio. Finalmente, en ningún caso se pretendió efectuar una comparación con el reemplazo valvular.

Características clínicas. En esta serie la mayoría de los pacientes estaba cursando una endocarditis activa, a diferencia de otras publicaciones en que la mayoría de los casos corresponden a endocarditis inactiva ${ }^{9,21,24}$. La duración del tratamiento antibiótico preoperatorio fue en promedio de 3 semanas, si bien 50\% tenía 10 o menos días de tratamiento, lo que puede ser resultado de la aceptación de los criterios actuales de intervención quirúrgica precoz, para prevenir mayor destrucción valvular, extensión perivalvular $\mathrm{y}$ diseminación extracardíaca de la infección, situaciones todas que ensombrecen el pronóstico de la endocarditis en general y disminuyen la probabilidad reparación valvular en particular ${ }^{1,4,9,22,24}$.

Como en casi todas las series, la indicación quirúrgica fue en la mayoría de los casos hemodinámica, por manifestaciones de insuficiencia cardíaca, si bien la indicación puramente ecocardiográfica fue también frecuente ${ }^{1,4}$.

Bacteriología. Como en nuestra serie general, el germen más frecuente causante de la endocarditis fue el Streptococcus sp, en especial tipo viridans, lo que contrasta con series internacionales, en que el germen más común es el Staphylococcus aureus, para lo cual no tenemos una explicación clara 4 . Sin embargo, el germen no fue en ningún caso determinante para efectuar o no una reparación valvular.

Hallazgos y procedimientos quirúrgicos. Esta experiencia confirma que, en la actualidad, la presencia de una válvula aórtica bicúspide es el sustrato anatomopatológico más frecuentemente involucrado en la endocarditis aórtica y la degeneración mixomatosa en la endocarditis mitral, si bien la mayoría de estas últimas eran normales ${ }^{1,4,9}$. Por otra parte, de las 5 válvulas mitrales estructuralmente normales, 4 correspondieron a endocarditis secundaria a lesión de jet, por insufi- ciencia aórtica en válvula bicúspide, lo que de acuerdo a la experiencia de la Cleveland Clinic es el principal mecanismo de la endocarditis bivalvu$l^{2}{ }^{24}$. A diferencia de esta última experiencia, en que no hubo extensión de la endocarditis al esqueleto fibroso del corazón, en nuestra serie hubo 5 casos de abscesos perianulares ${ }^{24}$.

Las lesiones más frecuentes secundarias a la endocarditis propiamente tal fueron, tanto en válvula aórtica como mitral, la presencia de vegetaciones y las perforaciones de velos valvulares. Estas lesiones, así como las condiciones basales anormales de las válvulas en cuanto a degeneración mixomatosa mitral como malformación bicúspide aórtica, son susceptibles de procedimientos reparadores que permiten preservar la estructura valvular, como se demuestra en esta experiencia $y$ en la literatura internacional 1,5,6,9,14,16,21,23,24. Sin embargo, en esta última, las comunicaciones de reparaciones valvulares por endocarditis aórticas son prácticamente anecdóticas.

$\mathrm{Si}$ bien una de las ventajas de la reparación valvular es evitar el implante de material protésico, en esta serie se efectuó una anuloplastia mitral con anillo protésico en 6 casos, puesto que se consideró que remodelar y reforzar el anillo mitral era de mayor importancia que el riesgo de recidiva de la endocarditis. Además, a diferencia del reemplazo valvular en que el anillo de la prótesis es introducido en el anillo mitral, el anillo de Carpentier-Edwards va sobrepuesto a este último y rápidamente se epiteliza, por lo que, por lo menos teóricamente, sería menos susceptible a una reinfección ${ }^{23}$. En caso de producirse esta última sería factible efectuar, como único procedimiento, la remoción del anillo protésico.

Mortalidad y morbilidad. En esta experiencia no hubo mortalidad perioperatoria. Si bien ésta es una serie pequeña de pacientes, en cuanto a endocarditis mitral, series más numerosas, como la de la Cleveland Clinic, presentan una baja mortalidad global, de 2,9\%, siendo de 3,8\% para los casos de endocarditis activa ${ }^{9}$. En esta última situación se observó, además, una diferencia significativa con el reemplazo mitral, aunque estos últimos pacientes eran más graves. También en esa serie las complicaciones postoperatorias, principalmente cardiovasculares, respiratorias y neu- 
rológicas, fueron frecuentes, a diferencia de la nuestra ${ }^{9}$.

En cuanto a cirugía reparadora de la endocarditis aórtica, como se señaló, las comunicaciones en la literatura son anecdóticas, por lo que no es posible efectuar comparaciones. Como quiera que sea, la mortalidad para todos los pacientes con endocarditis infecciosa de válvula nativa operados en nuestra institución entre 1988 y 1999 fue de 6\%20.

Seguimiento. Si bien el promedio de seguimiento fue sólo algo mayor a 3 años, es destacable que no hubo mortalidad ni morbilidad relacionadas a las válvulas cardíacas, con excepción de un caso de recidiva de endocarditis aórtica, en un paciente con aplasia medular secundaria. En la serie de la Cleveland Clinic para endocarditis mitral la probabilidad de sobrevida libre de eventos a 6 años fue significativamente mayor para la reparación $(75 \%)$ que para el reemplazo $(30 \%)^{9}$. En cuanto a la

\section{REFERENCIAS}

1. Ferguson E, Reardon MJ, Letsou GU. The surgical management of bacterial valvular endocarditis. Curr Opin Cardiol 2000; 15: 82-5.

2. Grover FL, Cohen DJ, Oprian C, Henderson WG, Sethi G, HammermeISTER KE. Determinants of the occurrence of and survival from prosthetic valve endocarditis: experience of the Veterans Affairs Cooperative Study on Valvular Heart Disease. J Thorac Cardiovasc Surg 1994; 108: 207-14.

3. Lewis BS, Agathangelou NE, Colsen PR, Antunes M, KINSLEY RH. Cardiac operation during active infective endocarditis. J Thorac Cardiovasc Sur 1982; 84: $579-84$

4. Mylonakis E, Calderwood SB. Infective endocarditis in adults. N Engl J Med 2001; 345: 1318-30.

5. Carpentier A. Cardiac valve surgery the French correction». J Thorac Cardiovasc Surg 1983; 86: 323-37.

6. Giwnov AM, Cosgrove DM, Blackstone EH, Díaz R, ARnold JH, LytLe BW ET al. Durability of mitral valve repair for degenerative disease. J Thorac Cardiovasc Surg 1998; 116: 734-43. experiencia nacional, en nuestra serie de endocarditis ya citada, la sobrevida a 5 años de los pacientes operados fue de $81 \%{ }^{20}$.

Finalmente, en esta serie hubo una importante mejoría sintomática de los pacientes lo que se asoció a buenos y estables resultados ecocardiográficos.

Conclusiones. La cirugía reparadora de la endocarditis, tanto aórtica como mitral, es factible de llevar a cabo aplicando variados procedimientos reconstructores, tanto de las lesiones valvulares basales como de las secundarias al proceso infeccioso, el cual debe ser macroscópicamente erradicado. La mortalidad y morbilidad operatoria es baja y los resultados alejados buenos y estables, sin los inconvenientes de los distintos tipos de reemplazos valvulares, por lo que la reparación valvular de la endocarditis es una excelente alternativa de tratamiento a estos clásicos procedimientos.

7. Petterson G, Tingleff J, Joyce FS. Treatment of aortic valve endocarditis with the Ross operation. Eur J Cardiothorac Surg 1998; 13: 678-84.

8. Sabik JF, Lytle BW, Blackstone EH, Marulo aG, Pettersson GB, Cosgrove DM. Aortic root replacement with cryopreserved allograft for prosthetic valve endocarditis. Ann Thorac Surg 2002; 74: 650-9.

9. Muehrcke DD, Cosgrove DM, Lytle BW, Taylor PC, Burgar AM, Durnwald CP et al. Is there an advantage to repairing infected mitral valves? Ann Thorac Surg 1997; 63: 1718-24.

10. Gimnov AM, Faber CN, Sabik JF, Pettersson C, GRIFFIN BP, GoRdon SM ET AL. Endocarditis after mitral valve repair. Ann Thorac Surg 2002; 73: 1813-6.

11. Zalaquett R. Cirugía reparadora de la insuficiencia valvular mitral. Rev Chil Cir 1994; 46: 127-36.

12. Zalaquett R, Morán S, Irarrázaval MJ, Maturana G, Braun S, Chamorro G et al. Reconstrucción valvular mitral. Cirugía reparadora de la insuficiencia valvular mitral con evaluación ecocardiográfica transesofágica intraoperatoria. Rev Chil Cardiol 1996; 15: 78-84. 
13. Zalaquett R, Chamorro G, Braun S, Howard M, IrarRázaval M, Morán S ET al. Cirugía reparadora de la insuficiencia aórtica. Rev Chil Cardiol 1998; 17: 145

14. Zalaquett R, Chamorro G, Braun S, Garrido L, Howard M, Morán S et al. Resultados alejados de la cirugía reconstructora de la insuficiencia mitral. Rev Méd Chile 1999; 127: 1093-100.

15. Zalaquett R, Garrido L, Morán S, Irarrázaval MJ, Becker P, Maturana G et al. Cirugía de la insuficiencia mitral isquémica. Rev Méd Chile 2002; 130: 9-16.

16. Fraser CD, Wang N, Mee RBB, Lytle B, Mc CARTHY PM, SaPp SK et al. Repair of insufficient bicuspid aortic valves. Ann Thorac Surg 1994; 58: 386-90.

17. Von Reyn CF, Levy BS, Arbeit RD, Friedland G, CRUMPACKER CS. Infective endocarditis: an analysis based on strict case defenition. Ann Intern Med 1981; 94: 508-18.

18. DuRACK DT, LuKes AS, BRIChT DK. New criteria for diagnosis of infective endocarditis: Utilization of specific echocardiographic findings. Duke endocarditis service. Am J Med 1994; 96: 200-9.
19. Morán S, Larraín E, Braun S, Irarrázaval MJ, Dubernet J, Maturana G et al. Tratamiento quirúrgico de la endocarditis infecciosa activa. Rev Méd Chile 1988; 116: 882-6.

20. Braun S, Escalona A, Chamorro G, Corbalán R, Pérez C, Labarca J et al. Endocarditis infecciosa: análisis de 261 casos y resultados del tratamiento con un enfoque multidisciplinario. Rev Méd Chile 2000; 128: 708-20.

21. Hendren WG, Morris AS, Rosenkranz ER, Lytle BW, TAY LOR PC, SteWARt WJ ET aL. Mitral valve repair for bacterial endocarditis. J Thorac Cardiovasc Surg 1992; 103: 124-9.

22. Pettersson G, Carbon C, and The Endocarditis Working Group of the International Society of CHEMOTHERAPY. Recommendations for the surgical treatment of endocarditis. Clin Microbiol Infect 1998; 4: 3534-46.

23. Dreyfus G, Serraf A, Jebara VA, Deloche A, Chauvaud S, Couetil JP et al. Valve repair in acute endocarditis. Ann Thorac Surg 1990; 49: 706-11.

24. Giwnov AM, Díaz R, Biackstone EH, Pettersson GB, SabiK JF, Lytie BW et al. Double Valve Endocarditis. Ann Thorac Surg 2001; 71: 1874-9. 